\title{
Imaging the cellular response to transient shear stress using time-resolved digital holography
}

\author{
Yoshihiko Arita $^{a}$, Maciej Antkowiak ${ }^{a, b}$, Frank Gunn-Moore $^{b}$ and Kishan Dholakia ${ }^{a}$ \\ ${ }^{a}$ SUPA, School of Physics and Astronomy, University of St Andrews, St Andrews, Fife, KY16 \\ 9SS, United Kingdom; \\ ${ }^{b}$ SULSA, School of Biology, Medical and Biological Sciences Building, University of St \\ Andrews, St Andrews, Fife, KY16 9TF, United Kingdom
}

\begin{abstract}
Shear stress has been recognized as one of the biophysical methods by which to permeabilize plasma membranes of cells. In particular, high pressure transient hydrodynamic flows created by laser-induced cavitation have been shown to lead to the uptake of fluorophores and plasmid DNA. While the mechanism and dynamics of cavitation have been extensively studied using a variety of time-resolved imaging techniques, the cellular response to the cavitation bubble and cavitation induced transient hydrodynamic flows has never been shown in detail. We use time-resolved quantitative phase microscopy to study cellular response to laser-induced cavitation bubbles. Laser-induced breakdown of an optically trapped polystyrene nanoparticle (500 $\mathrm{nm}$ in diameter) irradiated with a single nanosecond laser pulse at $532 \mathrm{~nm}$ creates transient shear stress to surrounding cells without causing cell lysis. A bi-directional transient displacement of cytoplasm is observed during expansion and collapse of the cavitation bubble. In some cases, cell deformation is only observable at the microsecond time scale without any permanent change in cell shape or optical thickness. On a time scale of seconds, the cellular response to shear stress and cytoplasm deformation typically leads to retraction of the cellular edge most exposed to the flow, rounding of the cell body and, in some cases, loss of cellular dry mass. These results give a new insight into the cellular response to laser-induced shear stress and related plasma membrane permeabilization. This study also demonstrates that laser-induced breakdown of an optically trapped nanoparticle offers localized cavitation (70 $\mu \mathrm{m}$ in diameter), which interacts with a single cell.
\end{abstract}

Keywords: Digital holography, Time-resolved imaging, Laser-induced breakdown, Optical trapping, Cell transfection

\section{INTRODUCTION}

Laser-induced breakdown (LIB) causes a rapid temperature and pressure increase within the plasma followed by the expansion of a cavitation bubble accompanied by a high pressure hydrodynamic flow. During the collapse phase, a bubble close to the substrate forms hydrodynamic jets towards the surface. The subsequent outward flow along the surface collides with the inward moving bubble wall, ${ }^{1}$ which results in complex flow fields around the bubble. ${ }^{2}$ Such dynamical processes are central to a range of key techniques, particularly in the biomedical sciences including targeted cell lysis and dissection or inactivation of material such as cellular organelles, cytoskeletal filaments, and chromosomes. ${ }^{3-5}$ Such breakdown is also essential to the targeted delivery of genes or biomolecules via transient permeabilization of the plasma membrane of a cell (photoporation). ${ }^{2,6-10}$

In the past, time-resolved brightfield imaging with nanosecond temporal resolution was used to visualize the lysis zone and cellular deformation under the bubble. ${ }^{11}$ It was shown that the hydrodynamic shear flow from the expanding bubble is the main factor in cell deformation. ${ }^{12}$ The extent of cell lysis and molecular injection zones were quantitatively related to laser parameters and the created hydrodynamic pressure. ${ }^{6}$ In a similar manner, brightfield time-resolved imaging was employed to visualize cavitation dynamics in a microfluidics channel ${ }^{5}$ and in the rat cornea. ${ }^{13}$ Also, a fast camera with microsecond temporal resolution was used to quantify red blood

Further author information: (Send correspondence to Y.A.)

Y.A.: E-mail: ya10@st-andrews.ac.uk, Telephone: +44 (0)1334 461656

Optical Elastography and Tissue Biomechanics, edited by Kirill V. Larin,

David D. Sampson, Proc. of SPIE Vol. 8946, 89460V · (C) 2014 SPIE

CCC code: $1605-7422 / 14 / \$ 18 \cdot$ doi: $10.1117 / 12.2039973$

Proc. of SPIE Vol. 894689460 -1 
cell deformation induced by LIB of water. ${ }^{14}$ In all these studies, based on brightfield microscopy, the acquired images displayed only the deformation of the cell outline without any more detailed and quantifiable information about the actual changes within the cell's cytoplasm.

Time-resolved pulsed interferometry, realized both with femtosecond and nanosecond lasers, was previously used to study fast transient processes in various materials upon laser irradiation ${ }^{15}$ and ablation. ${ }^{16,17}$ Typically only a single interferogram at a predefined time delay is acquired although a multi-exposure modification was also proposed. ${ }^{18}$ Digital holography microscopy (DHM) at a video frame rate has been successfully used to quantify cellular response to focused nanosecond ${ }^{19}$ and femtosecond ${ }^{20}$ laser mediated cellular membrane poration. To our knowledge, time-resolved interferometry or quantitative phase imaging has never been used to study fast transient processes in live cells or tissues.

We recently proposed a new technique to create well localized cavitation bubbles ${ }^{2,7-9}$ that can be used to permeabilize the cellular membrane and introduce plasmid DNA into a small population of surrounding cells. In this approach, a nanosecond laser is focused onto a single nanoparticle, which is optically trapped in the proximity of the cells of interest. The breakdown threshold energy is significantly decreased when compared to the LIB of a liquid, providing a more controllable and confined cavitation. The total energy of the system is typically several orders of magnitude lower than that of water leading to a lower magnitude of hydrodynamic shear stress. As a result, no cell lysis zone is observed, with the majority of cells remaining viable, which makes this technique particularly suitable for single cell studies.

In this letter, we employ time-resolved quantitative phase imaging to gain a better insight into the transient cellular deformation under conditions of shear stress created by the LIB of a single nanoparticle. The combined implementation of off-axis DHM in a Mach-Zehnder geometry with either pulsed (nanosecond) or CW illumination enables us to study dynamics of the cellular response to transient shear stress at time scales spanning from microseconds to minutes. This versatile approach allows us not only to observe the deformation and displacement of the cell body but importantly to relate the long term cellular response to the initial reversible deformation of the cell body which is observable only at the microsecond scale.

\section{MATERIALS AND METHODS}

\subsection{Laser trapping and laser-induced breakdown}

A $1070 \mathrm{~nm}$ CW fiber laser (IPG Laser GmbH, YLM-5-1070-LP: power of $\sim 80 \mathrm{~mW}$ ) was focused by a microscope objective (Nikon Ltd., E plan $100 \times 1.25 \mathrm{NA} /$ oil) to optically trap a polystyrene nanoparticle (500 nm diameter, 3500A Thermo Fisher Scientific Inc.) within an aqueous medium, as shown in Fig. 1 (inset). The nanoparticle was held $15 \mu \mathrm{m}$ above the substrate. A single pulse from a $532 \mathrm{~nm}$ frequency doubled Q-switched Nd:YAG laser (Elforlight Ltd., SPOT: pulse width of $\sim 1 \mathrm{ns,} \mathrm{energy} \mathrm{of} \sim 1 \mu \mathrm{J}$ ) was co-aligned with the trapping beam and focused through the same objective onto the trapped nanoparticle.

\subsection{Digital holography microscopy}

Quantitative phase imaging was realized in a typical fiber based off-axis DHM setup. ${ }^{20}$ For time-lapse recordings, light from a laser diode (Hitachi HL6344G, $\lambda=635 \mathrm{~nm}$ ) was coupled into a single mode optical fiber splitter. Alternatively, in the nanosecond time-resolved imaging a second $532 \mathrm{~nm}$ Nd:YAG laser (Elforlight Ltd., SPOT: pulse width of $\sim 1 \mathrm{~ns}$, energy of $\leq 1 \mu \mathrm{J}$ ) was used in place of the CW laser diode. In that case both ns lasers were triggered by a delay pulse generator (Directed Energy, Inc., PDG-2515) where the illumination laser was triggered at a desired time delay $(50 \mathrm{~ns}-20 \mu \mathrm{s})$ after the LIB event. In both types of experiments a $0.5 \times$ lens between the microscope and the camera was used to demagnify the image giving the effective magnification of $50 \times$. Off-axis holograms were recorded by a CCD camera (Imaging Source, DMK31BU03) running either at the full frame rate of $25 \mathrm{fps}$ or triggered for single frame time-resolved imaging. In the latter case one stroboscopic hologram was captured for each triggered LIB event. In addition, a hologram was acquired 1 second before and after the breakdown event. The complex wavefront in the object plane was reconstructed from the off-axis holograms using a Fourier space filtering technique either in real time using LabView or in MATLAB post-processing. When necessary the reconstructed phase was unwrapped and the quadratic curvature of the background was fitted and subtracted. 


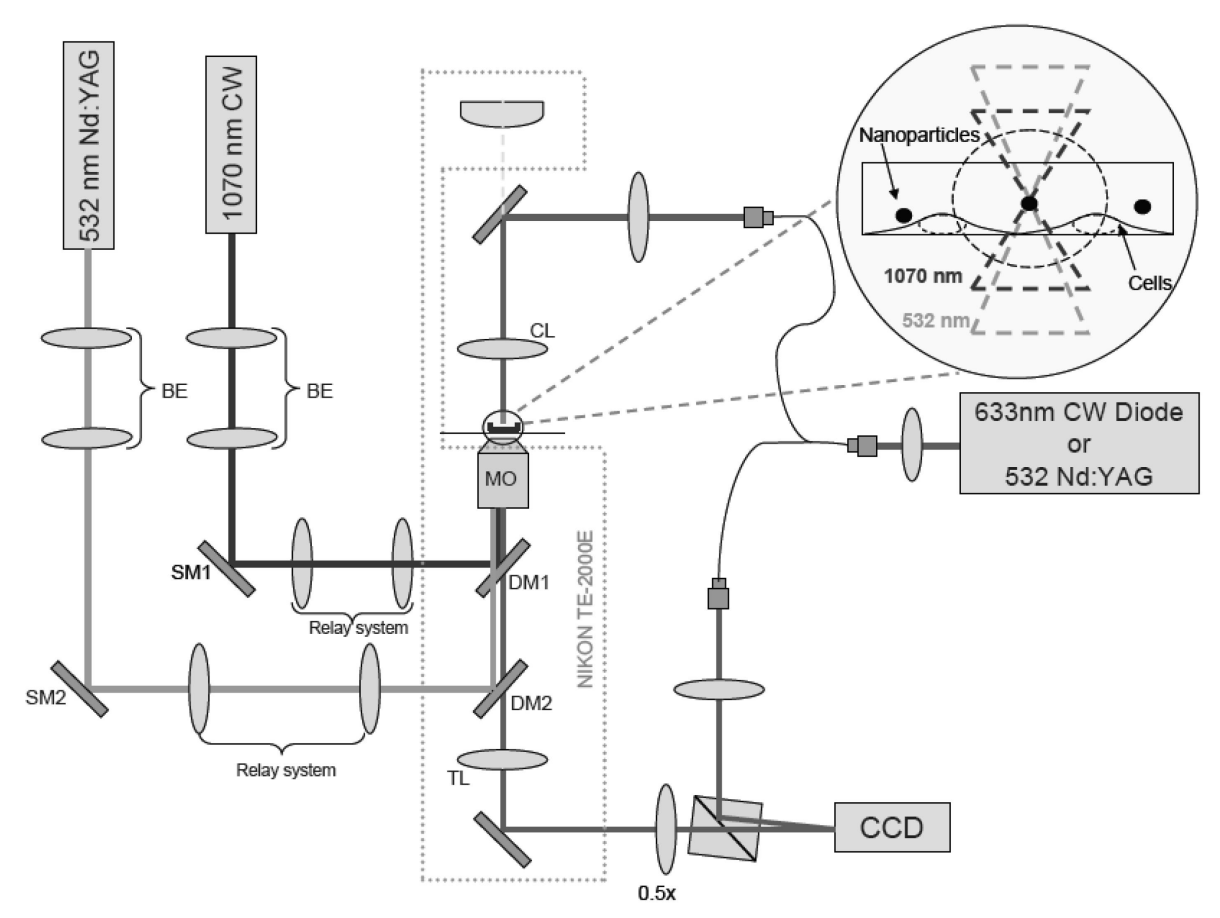

Figure 1. Experimental Setup. LIB and trapping beams are coupled into the microscope using dichroic mirrors DM1 (R80\%@1070 nm/T50\%@532 nm) and DM2 (R92\%@532 nm/T70\%@1070 nm). SM1 and SM2 are steering mirrors for the trapping and LIB beams, respectively.

\subsection{Cell preparation}

Chinese hamster ovary cells (CHO-K1) were used in the study. Cells were seeded onto glass-bottomed Petri dishes (World Precision Instruments) 48 hours prior to experiments and incubated at $37^{\circ} \mathrm{C}$ and with $5 \% \mathrm{CO}_{2}$ to assure complete cell attachment. For the experiment the culture medium was replaced with OptiMEM@ (Invitrogen) containing nanoparticles.

\section{RESULTS AND DISCUSSIONS}

\subsection{Time-lapse digital holography microscopy}

Figure 2 shows three typical reactions of cells observed over a few minutes after the LIB of a trapped nanoparticle. Contrary to the previous study with the LIB of a liquid, ${ }^{11}$ we observed no cell lysis in the vicinity of a cavitation bubble. This can be attributed to the lower breakdown threshold of the nanoparticle, which in turn leads to a less violent cavitation event, hence a lower hydrodynamic pressure is created in the system. The most common response is the initial mechanical displacement of the part of the cell facing the cavitation bubble by a few micrometers away from the center of cavitation as shown in Fig. 2b (the dark pattern in the phase difference map). Typically, over the time of a few seconds, cells start to retract further and round up in response to the transient stress as shown by the frames taken at 1 and 2 minutes after the LIB event. This is also clearly visible in the time trace of cell area (Fig. 2e). Interestingly, in some cases the stress response starts to be well pronounced after $1-2$ seconds even when no initial noticeable cell deformation or displacement can be observed immediately after the LIB (Fig. 2f-j). This suggests a more subtle and transient form of cell interaction with the expanding bubble and hydrodynamic flow. Comparison of changes in optical thickness (Fig. 2b-d and Fig. 2g-i) as well as time traces of cell area (Fig. 2e and Fig. 2j) clearly show that the side of the cell facing the hydrodynamic flow is being quickly retracted and the cytoplasm from this part is transported towards the center of the cell leading to a gradual increase in thickness. At the same time no change in the cell footprint is observed in areas not directly facing the direction of the shear stress. The typical time scale of this reaction suggests that it is related 
to a local activation of shear stress response pathways leading to cytoskeleton reorganization. Importantly, no or minimal loss of total optical thickness, which is proportional to the total cellular dry mass ${ }^{21}$ is observed in these two cases (Fig. 2e and 2j).
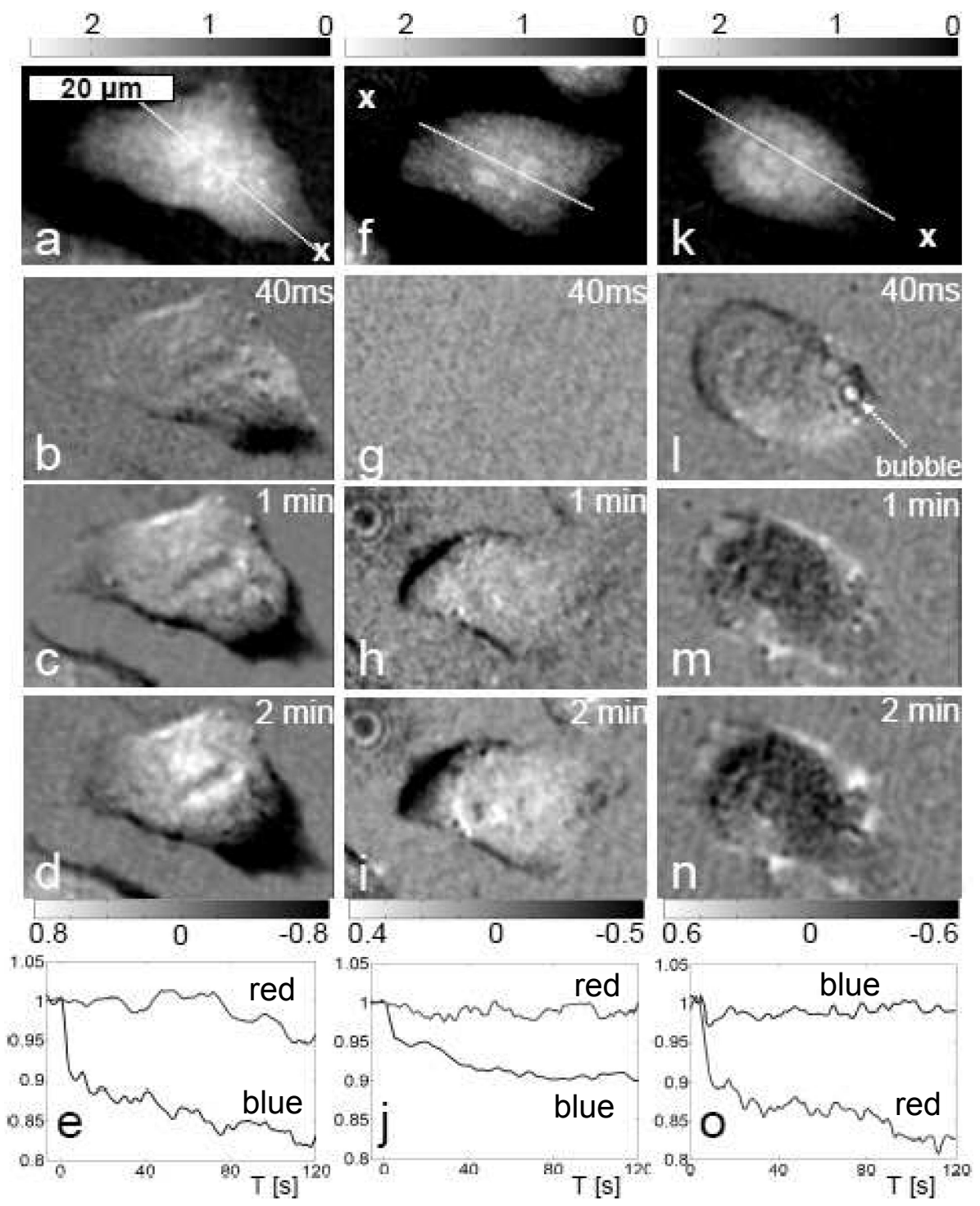

Figure 2. Phase maps of cellular reaction to transient shear stress. LIB sites are marked with ' $\mathrm{x}$ '. a, $\mathbf{f}$ and $\mathbf{k}$ show phase maps while all other images show the difference in phase between a given moment and the state before. Time traces in e, $\mathbf{j}$ and $\mathbf{o}$ show the respective cellular dry mass (red) and area (blue) normalized to the state before LIB. The breakdown occurs at time $T=0$. All phase maps in radians. Scale bar $20 \mu \mathrm{m}$ common to all figures.

In rare cases a significant gradual loss of dry mass is observed as shown in Fig. 2k-o. This type of response strongly correlates with appearance of long lasting $(<0.5 \mathrm{~s})$ residual gas bubbles on the cellular membrane

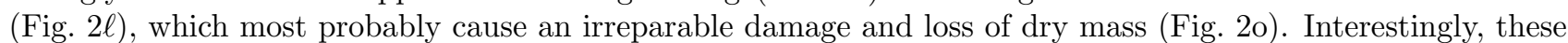
bubbles do not appear in all cases. Moreover, they are typically localized on the cell membrane rather than in the center of cavitation. The formation of residual gas bubbles was also previously reported by Rau et al. in the LIB of a liquid. ${ }^{12}$ 


\subsection{Time-resolved digital holography microscopy}

The cavitation dynamics and transient deformation of the cell body under hydrodynamic shear stress can be revealed in detail by the time-resolved DHM. Importantly, it also enables a direct measurement of the evolution of the cavitation. The produced cavitation bubbles had a typical maximum radius of $r \sim 35 \mu \mathrm{m}$ with a lifetime of $t \sim 12 \mu \mathrm{s}$. Notably, the presence of a monolayer of cells affects the cavitation dynamics, in which case the maximum radius is smaller than that without cells. ${ }^{12}$ In the LIB of a nanoparticle the maximum extent of the bubble is typically limited to the first circle of surrounding cells. It appears that a significant part of the bubble energy is absorbed by these cells leading to a very strong transient deformation. Based on a detailed time-resolved imaging of cavitation bubble evolution ${ }^{2}$ and using the numerical model proposed by Rau et al., ${ }^{12}$ we can estimate the maximum shear stress related to the radial hydrodynamic flow to be on the order of $10-100 \mathrm{kPa}$.

Figure 3 shows quantitative phase maps of the transient deformation of cells at $3 \mu \mathrm{s}$ (Fig 3a-d) and $15 \mu \mathrm{s}$ (Fig 3e-h) after the LIB of a nanoparticle. In the first case the bubble is in an expansion phase with the hydrodynamic flow directed away from the bubble. Fig. 3b shows that the hydrodynamic flow caused by the expanding bubble exerts enough shear stress to push the cell bodies away from the cavitation center with a typical displacement of $2-5 \mu \mathrm{m}$ visible in the profiles in Fig. 3d. Noticeably, these profiles also show that the optical thickness of the cell near the edge exposed to the flow is reduced by as much as $70-80 \%$ indicating that most of the cytoplasm is pushed towards the inside of the cell. Unfortunately, the steep edge of the cavitation bubble does not allow for phase reconstruction of the cells already encompassed by the bubble. Fig $3 \mathrm{f}$ shows cells $15 \mu \mathrm{s}$ after the LIB, in which case the cavitation bubble has fully collapsed. Noticeably, the cells are still deformed, and cell 1 (in Fig 3f) close to the cavitation center is still pushed away while cell 2 (in Fig 3f) at a larger distance from the center, is pulled towards it. This reveals for the first time that the hydrodynamic flow fields cause the cells to move back and forth during the bubble's expansion and collapse, which can enhance intracellular movement and related stress. As a result the cells experience a shear stress not only on the membrane but also within the cytoplasm. Importantly, in both cases all the cells return to their initial positions with no change in the footprint or optical thickness $1 \mathrm{~s}$ after the LIB event (Fig. 3c and 3g). This indicates that in some cases the produced shear stress from the LIB of a nanoparticle does not affect the adherence of the cells to the substrate and that the movement of the cytoplasm results only in a fully reversible elastic deformation of the cytoskeleton. This observation is consistent with the delayed cellular response without any initial deformation shown in Fig. 2f-j.
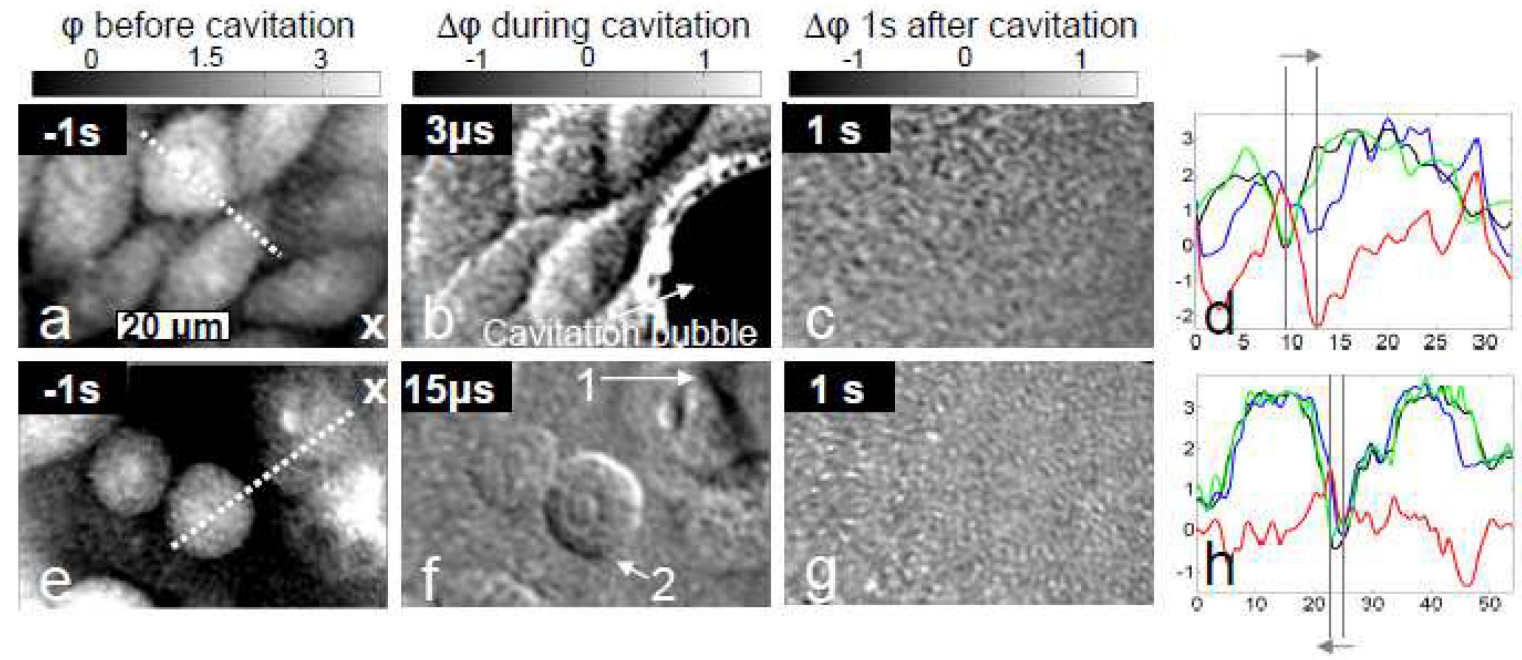

Figure 3. Phase maps of cellular deformation at different time delays after LIB. The nanoparticle positions are marked with ' $\mathrm{x}$ '. a and e show phase maps before LIB while all other images show the difference in phase between a given moment after LIB and the state before. Profiles in $\mathbf{d}$ and $\mathbf{h}$ show the cross-section through corresponding phase maps along lines shown in a and e respectively: the state before (black), during cavitation (blue), $1 \mathrm{~s}$ after cavitation (green); red curve shows the difference between the phase maps, during (blue) and before cavitation (black). All phase maps in radians. Scale bar $20 \mu \mathrm{m}$ common to all figures. 
Our findings revealed that both the cavitation bubble and the related hydrodynamic flow exert a considerable shear stress on the cell body leading to transient cytoplasm deformation. For the first time a bi-directional cytoplasm displacement was observed during expansion and collapse of the cavitation bubble. Noticeably, in some cases cell deformation was only observable at the microsecond timescale without any permanent change in cell shape or optical thickness. The comparison of cellular responses observed at different time scales suggests that in such cases the slow cellular reaction may be delayed by up to $1-2$ seconds, usually leading to retraction of the cellular edge most exposed to the flow.

We believe that the presented observations give a valuable insight into cell deformation and reaction to transient external shear stress. The time-resolved phase images suggest that the mechanism responsible for cellular membrane permeabilization and the previously reported plasmid DNA transfection ${ }^{2,7,8}$ is most probably a combination of a direct interaction with the expanding bubble for the first row of cells and with the transient hydrodynamic flow elsewhere. The presented time-resolved holographic technique in combination with the creation of controllable cavitation bubbles can be used in further single cell quantitative studies of cellular elastic deformation under transient shear stress providing a better understanding of the viscous and elastic properties of adherent cells. ${ }^{9}$

\section{REFERENCES}

[1] Tomita, Y. and Shima, A., "Mechanisms of impulsive pressure generation and damage pit formation by bubble collapse," J. Fluid Mech. 169, 535-564 (1986).

[2] Arita, Y., Antkowiak, M., Venugopalan, V., Gunn-Moore, F. J., and Dholakia, K., "Dynamics of primary and secondary microbubbles created by laser-induced breakdown of an optically trapped nanoparticle," Phys. Rev. E 85(1), 016319 (2012).

[3] Botvinick, E. L., Venugopalan, V., Shah, J. V., Liaw, L. H., and Berns, M. W., "Controlled ablation of microtubules using a picosecond laser," Biophys. J. 87(6), 4203-4212 (2004).

[4] Vogel, A., Lorenz, K., Horneffer, V., Huttmann, G., von Smolinski, D., and Geberty, A., "Mechanisms of laser-induced dissection and transport of histologic specimens," Biophys. J. 93(12), 4481-4500 (2007).

[5] Quinto-Su, P. A., Lai, H. H., Yoon, H. H., Sims, C. E., Allbritton, N. L., and Venugopalan, V., "Examination of laser microbeam cell lysis in a PDMS microfluidic channel using time-resolved imaging," Lab Chip 8(3), 408-414 (2008).

[6] Hellman, A. N., Rau, K. R., Yoon, H. H., and Venugopalan, V., "Biophysical response to pulsed laser microbeam-induced cell lysis and molecular delivery," J. Biophotonics 1(1), 24-35 (2008).

[7] Arita, Y., Torres-Mapa, M. L., Lee, W. M., Cizmar, T., Campbell, P., Gunn-Moore, F. J., and Dholakia, K., "Spatially optimized gene transfection by laser-induced breakdown of optically trapped nanoparticles," Appl. Phys. Lett. 98(9), 093702 (2011).

[8] Antkowiak, M., Arita, Y., Dholakia, K., and Gunn-Moore, F., "Imaging the cellular response to transient shear stress using stroboscopic digital holography," J. Biomed. Opt. 16(12), 120508 (2011).

[9] Arita, Y., Ploschner, M., Antkowiak, M., Gunn-Moore, F., and Dholakia, K., "Laser-induced breakdown of an optically trapped gold nanoparticle for single cell transfection," Opt. Lett. 38(17), 3402-3405 (2013).

[10] Compton, J. L., Hellman, A. N., and Venugopalan, V., "Hydrodynamic determinants of cell necrosis and molecular delivery produced by pulsed laser microbeam irradiation of adherent cells," Biophys. J. 105(9), 2221-2231 (2013).

[11] Rau, K. R., Guerra, A., Vogel, A., and Venugopalan, V., "Investigation of laser-induced cell lysis using time-resolved imaging," Appl. Phys. Lett. 84(15), 2940-2942 (2004).

[12] Rau, K. R., Quinto-Su, P. A., Hellman, A. N., and Venugopalan, V., "Pulsed laser microbeam-induced cell lysis: Time-resolved imaging and analysis of hydrodynamic effects," Biophys. J. 91(1), 317-329 (2006).

[13] Cherian, A. V. and Rau, K. R., "Pulsed-laser-induced damage in rat corneas: time-resolved imaging of physical effects and acute biological response," J. Biomed. Opt. 13(2), 024009 (2008).

[14] Quinto-Su, P. A., Kuss, C., Preiser, P. R., and Ohl, C. D., "Red blood cell rheology using single controlled laser-induced cavitation bubbles," Lab Chip 11(4), 672-678 (2011).

[15] Temnov, V. V., Sokolowski-Tinten, K., Zhou, P., and von der Linde, D., "Ultrafast imaging interferometry at femtosecond-laser-excited surfaces," J. Opt. Soc. Am. B 23(9), 1954-1964 (2006). 
[16] Furutani, H., Fukumura, H., and Masuhara, H., "Nanosecond time-resolved interferometric study on morphological dynamics of doped poly(methyl methacrylate) film upon laser-ablation," Appl. Phys. Lett. 65(26), 3413-3415 (1994).

[17] Balciunas, T., Melninkaitis, A., Tamosauskas, G., and Sirutkaitis, V., "Time-resolved off-axis digital holography for characterization of ultrafast phenomena in water," Opt. Lett. 33(1), 58-60 (2008).

[18] Liu, Z. W., Centurion, M., Panotopoulos, G., Hong, J., and Psaltis, D., "Holographic recording of fast events on a CCD camera," Opt. Lett. 27(1), 22-24 (2002).

[19] Yu, L. F., Mohanty, S., Zhang, J., Genc, S., Kim, M. K., Berns, M. W., and Chen, Z. P., "Digital holographic microscopy for quantitative cell dynamic evaluation during laser microsurgery," Opt. Express 17(14), 1203112038 (2009).

[20] Antkowiak, M., Torres-Mapa, M. L., Dholakia, K., and Gunn-Moore, F. J., "Quantitative phase study of the dynamic cellular response in femtosecond laser photoporation," Biomed. Opt. Express 1(2), 414-424 (2010).

[21] Popescu, G., Park, Y., Lue, N., Best-Popescu, C., Deflores, L., Dasari, R. R., Feld, M. S., and Badizadegan, K., "Optical imaging of cell mass and growth dynamics," Am. J. Physiol-Cell Ph. 295(2), C538-C544 (2008). 